\title{
生态学研究中常见的统计学问题分析
}

\author{
张 峰 ${ }^{1,2}$ 武玉珍 ${ }^{2}$ 张桂萍 ${ }^{2,3}$ 茹文明 2,3
}

（1 山西大学生命科学与技术学院, 太原 030006)

（2 山西大学黄土高原研究所, 太原 030006）（3 长治学院生化系, 山西长治 046000）

\begin{abstract}
摘 要 在当代生态学研究中统计学方法的应用日益广泛, 对于生态科学的发展和研究水平的提高起到了积极的 作用。但是不容忽视的是在生态学研究应用统计学方法的过程中存在若干问题, 主要表现在: 1) 回归分析方面的 问题。直线回归方程用相关指数 $R^{2}$ 来描述直线回归的显著性; 曲线回归方程往往用相关系数 $r$ 来表示显著性; 多 元线性回归方程只对方程进行显著性检验, 没有对每一个回归系数进行显著性检验。2)方差分析方面的问题。当 处理数超过 2 时, 不恰当地使用 $t$-检验比较平均数的差异显著性。该文分析了产生这些问题的原因, 提出了改进 的对策。
\end{abstract}

关键词 生态学研究 统计学应用 问题与错误

\section{ANALYSIS ON SOME MISTAKES IN THE APPLICATION OF STATISTICS IN ECOLOGICAL RESEARCHES}

\author{
ZHANG Feng ${ }^{1,2}$ WU Yu-Zhen ${ }^{2}$ ZHANG Gui-ping ${ }^{2,3}$ and RU Wen-ming ${ }^{2,3}$ \\ (1 School of Life Science and Technology, Shanxi University, Taiyuan 030006, China) \\ (2 Institute of Loess Plateau, Shanxi University, Taiyuan 030006, China) \\ (3 Department of Biology and Chemistry, Changzhi College, Changzhi, Shanxi 046000, China)
}

\begin{abstract}
The application of statistical methods in ecological researches is more and more extensive, and it is very important for improvement and development of ecologica science. However, there are some mistakes when statistical methods are applied to ecological researches, 1) Regression analysis: correlation index, $R^{2}$, was incorrectly used to test whether a simple regression equation was significant or not. Correlation coefficient, $r$, was inappropriately used to describe the significance of curve regression equations. Moreover, multiple linear regression equation was only tested by analysis of variance (ANOVA), but each regression coefficient in the equation was not tested; 2) ANOVA. $t$-test was applied inappropriately to test significance of the means of all treatments when the treatments in an experiment were more than 2 . The reasons for these mistakes were investigated and methods to avoid these mistakes were introduced.
\end{abstract}

Key words Ecological researches, Statistical methodology, Mistake

加拿大著名生态学家 Pielou 曾经指出“生态学 本质上是一门数学” (Pielou, 1985)。据统计, 从 $1990 \sim 2004$ 年我国《植物生态学报》共发表论文 1094 篇, 其中与统计学有关的论文 922 篇, 占 $84.28 \%$; 《生态学报》共发表论文 2062 篇, 其中与统 计学有关的论文 1687 篇, 占 $81.81 \%$ 。这充分说明 了统计学方法在当代生态学研究工作中的重要地位 和作用。

现在可以毫不夸张地说, 离开了统计学, 面对纷 繁复杂的生态学数据我们将束手无策, 难以处理这 些数据并从中获得科学性的结论, 揭示生态学的内 在规律, 解释生态学现象。然而在众多的生态学文
献中, 应用统计学理论和方法时存在许多问题, 甚至 不恰当的方法和结论, 认真分析和思考这些问题将 有助于我们正确应用统计学理论和方法, 减少犯错 误的可能性, 促进生态学研究水平的提高。本文对 目前生态学研究工作中应用统计学理论和方法等方 面存在的问题进行研究, 分析存在这些问题的根源, 并提出相应的对策, 以期引起生态学工作者的注意, 从而减少和避免这些问题, 起到抛砖引玉的作用。

\section{1 回归分析方面的问题与原因}

在多数生态学文献中, 回归分析方面的问题是 所有应用统计学中出现频率最高的问题。主要表现 
在以下几个方面。

\section{1 直线回归问题}

1.1.1 用 $R^{2}$ (相关指数)来描述直线回归的显著性 直线回归分析又称为一元线性回归分析, 是回 归分析中方法最简单，应用最广泛的方法。在某些 生态学文献中, 对于回归方程的检验和表述不正确, 常用相关指数 $R^{2}$ 来刻划回归方程的显著性(庞奖 励等, 2001; 孙谷畴等, 2004; 肖天和王荣, 2002)。需 要指出的是, 相关指数 $R^{2}$ 是用来刻画曲线回归方 程拟合程度的重要指标, 并非检验直线回归方程是 否显著的统计量 (方开泰等, 1988; 杜荣寒, 2003)。 尽管对于直线回归方程有 $R^{2}=r^{2}$, 但应用 $R^{2}$ 来表 示直线回归方程的显著性显然是不恰当的 (Janssen \& Heuberger, 1995)。

对于直线回归方程 $(\hat{Y}=a+b X)$ 的显著性检 验, 包括回归方程的方差分析, 回归系数 $a 、 b$ 的显 著性检验 $(t$-检验) 和相关系数 $(r)$ 的显著性检验等。 本质上, 上述 3 种显著性检验方法是等价的, 亦即回 归方程显著 $(p<0.05) \Leftrightarrow$ 回归系数显著 $\Leftrightarrow$ 相关系数 $(r)$ 显著(方开泰等, 1988; 杜荣骞, 2003)。

产生这一错误的主要原因是: 1) 作者没有弄清 楚直线回归分析与曲线回归分析的最根本区别是什 么。2)更常见的错误来源于对微软 Office 缺乏客观 的认知。作为 Office (Microsoft) 主要组成部分的 Ex$\operatorname{cel}$ (或 Word), 在应用它进行图表编辑的时候可以对 数据添加趋势线和回归方程, 对回归方程的显著性 用 $R^{2}$ 来表示。许多作者想当然认为 Microsoft 的软 件肯定不会有问题,所以这些错误屡见不鲜。

\subsection{2 回归关系与函数关系的问题}

对直线回归方程的准确表达式为: $\hat{Y}=a+b X$, 但是几乎所有的生态学文献中, 回归方程都写为: $Y$ $=a+b X$ (庞奖励等, 2001; 孙谷畴等, 2004; 张慧杰 等, 2000; 肖天和王荣, 2002)。比较这两个回归方 程区别在于前者 $Y$ 上边有“^”( 读作“帽”( Hat), “^” 在统计学中表示“估计”(Estimate) 的意思), 而后者 没有“^”。因为有“^”的存在, 就意味着它有误差, 那 么自变量与因变量之间就是回归方程 (关系)。如果 没有“へ”, 那变量之间就是函数关系, 而非回归(或相 关)关系。许多作者没有意识到这一细微的差别, 在 数学上却具有根本不同的意义, 类似的问题在曲线 回归和多元回归分析中也普遍存在。

\section{2 曲线回归问题}

曲线回归问题主要集中于曲线回归方程的检验 问题,往往用相关系数 $r$ 来表示方程的显著性(朴河
春等, 2001; 郑逢中等, 1992; 肖瑜, 1992)。

统计学上, 对于曲线回归问题往往是将对应的 曲线模型转化为线性模型, 然后按照直线回归的方 法求出回归方程。在直线回归中, 既可以用相关系 数对回归方程进行显著性检验, 也可以用方差分析 和 $t$-检验的方法分别对方程和回归系数 $b$ 进行显 著性检验。

许多作者仍然不清楚, 迄今为止对于曲线回归 方程仍然没有显著性检验的方法, 只能对曲线回归 方程的拟合程度进行判断(方开泰等, 1988; 杜荣寒, 2003)。常用的方法有剩余(失拟)平方和, $S S_{e}$,

$$
S S_{e}=\Sigma\left(Y_{i}-\hat{Y}_{i}\right)^{2}
$$

式 (1)中 $Y_{i}$ 为实际观测值, $\hat{Y}_{i}$ 为回归值。一般来说, $S S_{e}$ 越小表明曲线回归方程的拟合程度就越好; 反 之亦然。

$S S_{e}$ 是对曲线回归方程失拟程度的绝对度量, 但是对于不同的曲线回归方程, 用 $S S_{e}$ 难以比较它 们拟合程度的优劣。因此, 采用相关指数更为合理 和方便。相关指数的定义为:

$$
R^{2}=1-\frac{S S_{e}}{S_{Y Y}}
$$

式(2)中 $S_{Y Y}$ 为因变量 $Y$ 的离差平方和。 $R^{2}$ 实际上 是一个相对的度量, 它表示回归平方和占总平方和 的百分比。 $R^{2}$ 越大表明曲线回归方程的拟合程度 就越好; 反之亦然。

\section{3 多元线性回归方面的问题}

在多元线性回归分析中常见的错误是，只对回 归方程进行显著性检验, 而没有对每一个回归系数 进行显著性检验 (关文涁等, 2000; 曾希柏等, 2000; 程达武等,2001; 何园球等, 1992)。

多元线性回归方程一般形式如下:

$$
\hat{Y}=b_{0}+b_{1} X_{1}+b_{2} X_{2}+\cdots+b_{k} X_{k}
$$

式(3)中 $b_{0}, b_{1}, b_{2}, \cdots, b_{k}$ 是回归系数; $X_{1}, X_{2}, \cdots$, $X_{k}$ 分别是自变量。对于方程 (3) 必须进行显著性 检验, 以便确定 $Y$ 与 $X_{1}, X_{2}, X_{3}, \cdots$ 之间是否存在 线性关系。如果方程 $(3)$ 显著 $(p<0.05)$ 并不意味着 每个自变量 $X_{1}, X_{2}, X_{3}, \cdots$ 对 $Y$ 的影响都重要, 可 能有的自变量重要 $(p<0.05)$, 而有的自变量则可有 可无 $(p>0.05)$, 因此在对方程进行显著性检验的基 础上, 就必须对所有回归系数进行显著性检验(方开 泰等, 1988)。对于那些不显著的回归系数 $b_{i}$, 其对 应的自变量 $X_{i}$ 要从方程中剔除, 以确保每个回归系 数都显著。 


\section{2 方差分析方面的问题与原因}

在处理数超过 2 时, 仍然使用 $t$-检验来进行每 个处理平均数之间的比较 (祝心如和王大力, 1997; 史作民等, 1998）。从方法上讲, 两两平均数之间的 显著性检验可以应用 $t$-检验 (又称 $L S D$ (Least significant difference) 法), 但有致命的缺点: 1) 使犯 I 型错 误的概率大大增加, 2)计算较为烦琐。

假设有 3 个处理, 那么要比较的平均数对数为: $\mathrm{C}_{3}^{2}=3$ 对, 亦即 $\bar{x}_{1}-\bar{x}_{2}, \bar{x}_{1}-\bar{x}_{3}$ 和 $\bar{x}_{2}-\bar{x}_{3}$ 。按照假设 检验的理论和方法, 这 3 对平均数的 $t$-检验就有 3 个对应的零假设 $\left(\mathrm{H}_{0}\right)$ 和备择假设 $\left(\mathrm{H}_{\mathrm{A}}\right)$, 这里仅列出 3 个零假设: (1) $\mathrm{H}_{01}: \mu_{1}=\mu_{2} ;(2) \mathrm{H}_{02}: \mu_{1}=\mu_{3}$ 和 (3) $\mathrm{H}_{03}: \mu_{2}=\mu_{3}$ 。如果显著性水平: $\alpha=0.05$ (也称为犯 I 型错误的概率), 那么每个零假设成立的概率为: 1 $-\alpha=0.95$ 。由于 $\mathrm{H}_{01}, \mathrm{H}_{02}$ 和 $\mathrm{H}_{03}$ 是相互独立的, 要使 这 3 个零假设同时成立, 那么它的概率就等于 $(1-\alpha)^{3}=0.95^{3}=0.8574$ ( 按照独立事件的概率等 于每个事件概率的乘积法则)。此时, 犯 I 型错误的 概率: $\alpha^{\prime}=1-0.8574=0.1426$, 几乎是 0.05 的 3 倍。随着处理数的增加, 犯 I 型错误的概率呈几何 级数迅速增加。因此, 当处理数超过 2 时, 必须用方 差分析 ( $F$ 检验) 来检验各处理之间的差异是否显 著, 以避免犯 I 型错误概率的迅速增加 (Glover \& Mitchell, 1998)。当 $F>F_{0.05}$ 时, 进一步用多重比较 的方法来检验各处理平均数之间的差异是否显著, 使我们对各个平均数的差异性有更准确的了解。

\section{3 讨 论}

对于生态学家来说, 应用统计学方法处理实验 数据, 获得统计学结论, 分析生态学现象, 揭示生态 学的内在规律, 是当代生态学研究的常用手段之一。 正确应用统计学方法和理论, 有助于提高研究工作 的水平, 从中发现某些生态学规律, 可使研究结果和 结论经得起推敲和时间的检验。因此, 熟悉统计学 理论和方法是我们减少犯错误的前提和必然。

由于现在有多种统计学软件, 包括国外的 SAS、 SPSS、Minitab 和国产的 DPS 等, 而且都有相关的使 用手册 (说明), 使用起来非常方便。因此, 计算问题 已经彻底解决。但是我们应该清楚地认识到统计学 软件只能提供统计学方法的计算技术和数据的分析 结果, 针对具体的实验数据究竟应用那种方法正确、 合理、科学、得当则取决于我们对统计学原理和方法 的认知程度。
对于生态学工作者来说, 首先是要熟悉和掌握 统计学的基本理论和方法。需要指出的是掌握统计 学的理论和方法, 并不是要掌握统计学理论的具体 推导过程和步骤, 而是要知道每种方法的基本原理 和理论基础、应用范围、有哪些优缺点和对数据有什 么要求等。其次才是掌握如何使用这些现成的统计 学软件。实际上, 在上述相关程序使用的手册中, 不 仅给读者提供了如何使用计算机软件完成有关的统 计分析, 而且对涉及的统计学原理也有一定的介绍, 只要我们认真阅读这些内容, 就可以最大程度地避 免某些错误。此外, 关于这方面的内容在任何一本 生物统计学专著和教材中都有较为详细的说明和论 述。在对某些方法应用把握不准的情况下, 只要动 手查阅一下有关的专著和教材, 这方面的问题就会 迎刃而解。

\section{参 考 文 献}

Cheng DW(程达武), Zhang XR(张秀荣), Liu JH(刘建宏), Zhan JR (战景仁), Dong GL(董广林) (2001). Effects of temperature of the development, food-take, reproduction of Lema dilversa Baly. Acta Ecologica Sinica (生态学报),21, 498 501. (in Chinese with English abstract)

Du RQ(杜荣寒) (2003). Biostatistics (生物统计学). Higher Education Press, Beijing. (in Chinese)

Fang KT(方开泰), Quan H(全辉), Chen QY(陈庆余) (1998). Practical Regression Analysis (实用回归分析). Science Press, Beijing. 84,151. (in Chinese)

Glover TJ, Mitchell KJ (1998). An Introduction to Biostatistics. McGraw-Hill Companies, Inc. New York, 193 - 194.

Guan WB (关文涁), Zeng DH(曾德慧), Jiang FQ (姜风歧) (2000). Ecological studies on the relationship between the process of desertization and vegetation dynamics in the west of northern China: community diversity and desertization process. Acta Ecologica Sinica (生态学报), 21,93-98. (in Chinese with English abstract)

He YQ(何园球), Wang MZ(王明珠), Zhao QG (赵其国) (1992) . Effect of precipitation on chemical element migration in soil of tropical monsoon forest and rubber plantation. Acta Phytoecologica et Geobotanica Sinica (植物生态学与地植物学学 报), 16,266 - 275. (in Chinese with English Abstract)

Janssen PHM, Heuberger PSC (1995). Calibration of process-oriented models. Ecological Modelling, 83,55-66.

Pang JL (庞奖励), Huang CC (黄春长), Sun GN (孙根年) (2001). Heavy metal content of sewage irrigated soil in $\mathrm{Xi}$ ' an and its influence to tomato. Soil and Environment Sciences (土壤 与环境) , 10, 94-97. (in Chinese with English abstract)

Piao HC(朴河春), Liu QM(刘启明), Guo JH(郭景恒)，Yu DL (余登利), Ran JC (再景丞) ( 2001). Origins of soil organic carbon with the method of natural ${ }^{13} \mathrm{C}$ abundance in maize fields. 
Acta Ecologica Sinica (生态学报), 3,434-439. (in Chinese with English abstract)

Pielou EC (1985). Mathematical Ecology, 2nd edn. Wiely-Interscience, New York.

Shi ZM (史作民), Liu SR (刘世荣), Cheng RM (程瑞梅) (1998). Changes in plant species diversity in a restoration sequence of Quercus variabilis forest stands in Bao Tianman Mountain. Acta Phytoecologica et Geobotanica Sinica (植物生态学与 地植物学学报), 21, 415-421. (in Chinese with English abstract)

Sun GC(孙谷畴), Zhao P(赵平), Zeng XP(曾小平) (2004). Photosynthetic acclimation to growth-irradiance in two tree species of Magnoliaceae. Acta Ecologica Sinica (生态学报), 24, 1110 - 1117. (in Chinese with English abstract)

Xiao T (肖天), Wang R (王荣) (2002). Distribution of Sybechococcus in the Bohai Sea in autumn and spring. Acta Ecologica Sinica (生态学报), 22,2071-2078. (in Chinese with English abstract)

Xiao Y(肖瑜) (1992). Biomass and productive by natural Pinus henryi. Acta Phytoecologica et Geobotanica Sinica (植物生态学 与地植物学学报), 16,227 - 233. (in Chinese with English abstract)

Zeng XB(曾希柏), Hou GJ(侯光炣), Qing CL(青长乐), Xie DT (谢德体) ( 2000). Interaction of light and nitrogen in soilplant system. Acta Ecologica Sinica (生态学报), 21, 103 108. (in Chinese with English abstract)

Zhang HJ(张慧杰), Li JS(李建社), Zhang LP(张丽萍), Liang YH(梁岩华) (2000). Evolution on the host plant species and fitness to vegetable leafminer and its harmfulness. Acta Ecologica Sinica (生态学报), 20,134 - 138. (in Chinese with English abstract)

Zheng FZ(郑逢中), Lin P (林鹏), Zheng WJ(郑文教), Zhuang ZX(庄峙厦) (1992). Study on the absorption and removal of Kandelia candel for pollutant cadmium. Acta Phytoecologica et Geobotanica Sinica (植物生态学与地植物学学报), 16,220 226. (in Chinese with English abstract)

Zhu XR(祝心如), Wang DL(王大力) (1997). Potential effect of extracts of roots of Malus pumila and Populus canadensis on wheat growth. Acta Phytoecologica et Geobotanica Sinica (植物生 态学与地植物学学报), 21, 226-233. (in Chinese with English abstract) 